\title{
Surgical Malignancy Presenting as Gynecological Emergency
}

\author{
Prabha Lal ${ }^{1}$, Kiran Aggarwal ${ }^{1}$, Abha Singh ${ }^{1}$, Romesh Lal ${ }^{2}$
}

Departments of ${ }^{1}$ Obstetrics \& Gynaecology and ${ }^{2}$ Surgery, Lady Hardinge Medical College and Smt. Sucheta Kriplani Hospital, New Delhi-110001, India.

\section{Corresponding Author:}

Dr. Prabha Lal

Email id:drprabhalal@gmail.com

This is an Open Access article distributed under the terms of the Creative Commons Attribution License (creativecommons.org/ licenses/by/3.0).

\begin{abstract}
Background: Krukenberg tumors, due to ovarian torsion have been rarely reported. Case Report: A 35 year old lady presented to the surgical emergency with severe left sided lower abdominal pain. Abdominal examination revealed a $15 \times 10 \mathrm{~cm}$, tender, mobile mass arising from pelvis. An emergency ultrasound revealed twisted ovarian mass. Emergency laparotomy showed sigmoid colon mass along with bilateral ovarian masses with torsion and rupture of right side ovarian mass. Total abdominal hysterectomy with bilateral salpingo-ophorectomy with segmental resection of sigmoid colon with loop transverse colostomy and para-aortic lymphadenectomy was done. Histopathology confirmed the presence of adenocarcinoma of colon with Krukenberg's tumor. Conclusion: Krukenberg tumor must also be considered for differential diagnosis of ovarian torsion.
\end{abstract}

Keywords: Abdominal Pain, Colostomy, Krukenberg Tumor, Laparotomy, Ovarian Neoplasms.

\section{Introduction}

Krukenberg tumor is a metastatic signet ring adenocarcinoma of ovary with primary malignancy elsewhere in gastrointestinal tract. Stomach is the most common primary site, rarely origin can be from breast, thyroid, kidney, lung, endometrium and biliary tract. It is a rare tumor accounting for $1-2 \%$ of all ovarian malignancies $[1,2]$. It is usually a bilateral tumor and is difficult to differentiate from primary tumor. Here we report a case of Krukenberg tumor which had an unusual presentation.

\section{Case Report}

A 35 year old $\mathrm{P}_{2} \mathrm{~L}_{2} \mathrm{~A}_{1}$ presented to the gynaecology emergency with complaints of sensation of abdominal distension and mass per abdomen since two weeks and acute onset colicky lower abdominal pain of one week duration. She had no fever, bowel or bladder, menstrual or other gynaecological complaints. Last menstrual period was 13 days prior to date of admission. Her first pregnancy 7 years ago was a normal vaginal delivery. In the second pregnancy, she underwent an emergency LSCS for fetal distress. She had past history of combined oral contraceptive pills use for 18 months. There was no family history of any malignancy.

On examination, general condition was fair and vitals were stable. Systemic examination including respiratory and cardiovascular system was essentially normal. Abdomen was distended and a $15 \times 10 \mathrm{~cm}$ size well defined firm mass was felt arising from pelvis and extending supraumbilically. The mass was tender but freely mobile. Bowel sounds were present. On per speculum examination, cervix was hyper-trophied, vagina was healthy looking with minimal mucoid vaginal discharge. On per-vaginum examination same mass was felt through anterior and lateral fornices, uterus was anteverted and normal in size and felt separate from the mass. Another mass was felt in the pelvis through the pouch of Douglas which 
was fixed and non-tender. There were no nodules in the pouch of Douglas. Per rectal examination confirmed the vaginal findings. Rectal mucosa was found to be freely mobile.

An urgent ultrasound was performed which reported a right complex ovarian cyst $(15 \times 12 \times$ $8 \mathrm{~cm})$ with internal septations and reduced vascularity in the pedicle suggestive of torsion. Another similar mass $(8 \times 5.2 \mathrm{~cm})$ was seen in the left adnexa. Uterus was normal in size and endometrial thickness was $4.7 \mathrm{~mm}$. Free fluid was seen in peritoneal cavity. Upper abdomen was unremarkable. Her relevant workup up till now included a negative Pap's smear for intra-epithelial abnormality. Blood results showed CA 125: 277 U/L, hCG: 0.53 mIU/L, AFP: 2.36 U/L, CEA: 73.3 $\mathrm{U} / \mathrm{L}$. A presumptive diagnosis of bilateral ovarian masses (likely malignant) with clinical suspicion of either torsion or hemorrhage was made. Patient was prepared for exploratory laparotomy.

Per-operative findings revealed presence of edematous parieties with thickened peritoneum and multiple widespread peritoneal and intestinal deposits. Approximately 1.5 litres intra-peritoneal blood was seen oozing intermittently from a small rent in the posterior aspect of a $15 \times 10 \mathrm{~cm}$ bosselated twisted right ovarian mass with irregular surface with no excrescences. Left ovary was enlarged $(6 \times 6 \mathrm{~cm})$ and irregular with an intact capsule and no surface excrescences. Uterus and bilateral fallopian tubes were normal. Systematic bowel exploration was done. An approximately 2 $\mathrm{cm}$ constricting growth was seen in the sigmoid colon. Disseminated metastasis some measuring $>2 \mathrm{~cm}$ was found in peritoneum, omentum and bowel. Para-aortic lymph nodes were also involved. Bilateral paracolic gutters, liver, spleen, bladder and under surface of diaphragm were examined and found free of metastasis. A provisional diagnosis of sigmoid colon carcinoma stage IVA with Krukenberg's tumor with right sided ovarian torsion with tumor bleed was made. Total abdominal hysterectomy with bilateral salphingo-

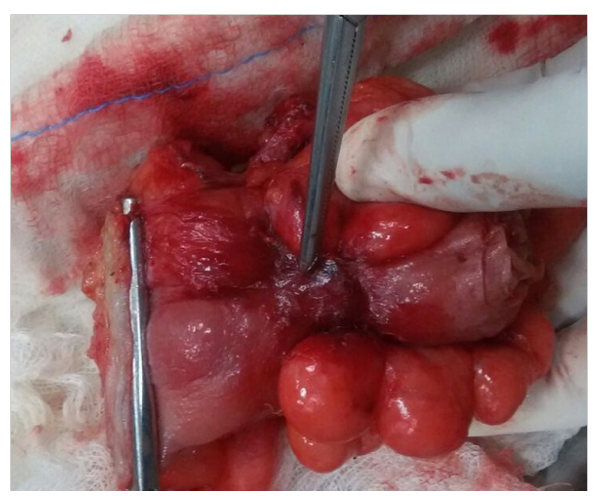

Fig.1: Constricting primary sigmoid colon carcinoma.

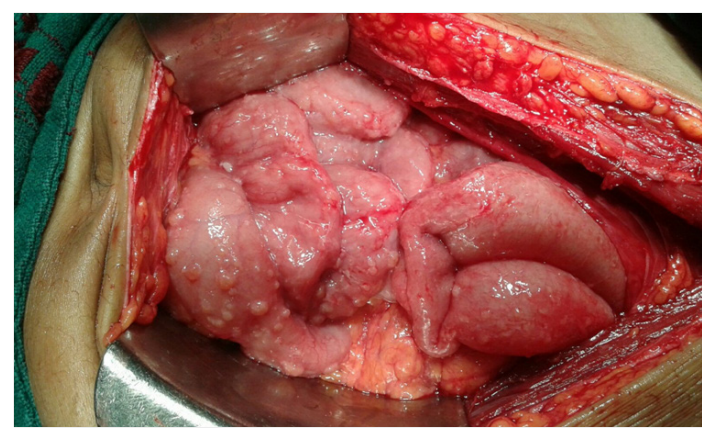

Fig.2: Metastatic lesions in small and large intestine.

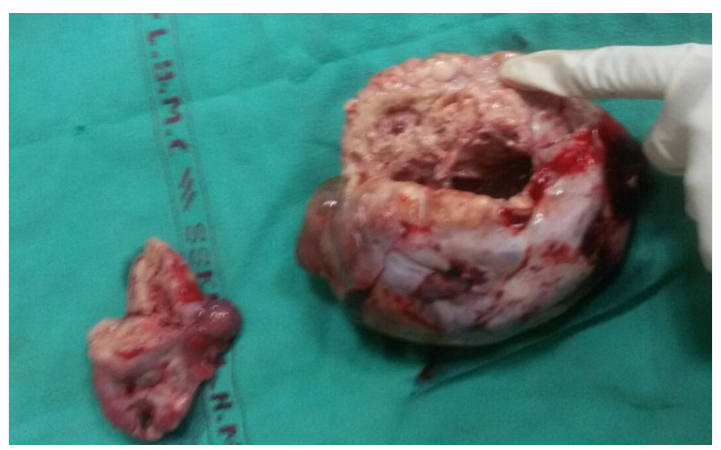

Fig.3: Excised portion of sigmoid colon showing constricting growth.

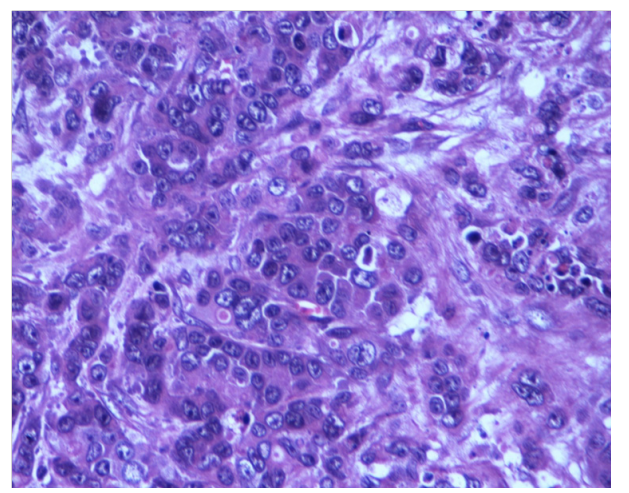

Fig.4: Ovary showing inconspicuous signet ring cells. 
oophorectomy with infracolic omentectomy and para-aortic lymphadenectomy was performed. Segmental resection of sigmoid colon was done along with its lymphatic drainage area with a loop colostomy. Three units packed red blood cells were transfused per-operatively. CECT scan of chest and abdomen done on $5^{\text {th }}$ post-operative day did not show any evidence of metastasis. The postoperative period was uneventful. The patient was discharged on the $10^{\text {th }}$ post-operative day.

The histopathology report of ovaries revealed presence of inconspicuous signet ring cells whereas excised tissue revealed low grade adenocarcinoma colon. There was evidence of metastasis to both ovaries, omentum and paraaortic nodes with pathological staging of $\mathrm{T} 4 \mathrm{~b}$ PN1a. Patient was given 10 cycles of chemotherapy with FOLFOX regime after three weeks of surgery. However patient initially did well and expired 15 months following surgery.

\section{Discussion}

Krukenberg tumor was first reported by a German gynecologist Fredrick in 1896 as a new type of primary malignant ovarian tumor but later R. H Major revealed the true metastatic nature of the lesion. It is a rare metastatic signet ring cell tumor and accounts for $1-2 \%$ of allovarian tumors [1]. Majority of the cases are synchronous, but 20-30\% cases occur as metachronous lesions after removal of primary tumor [1,2]. Most common primary site for Krukenberg tumor is stomach and majority spread by retrograde lymphatics. Less commonly it may arise from colon, biliary tract, appendix, breast and gall bladder. Primary tumor may not be found in $10 \%$ of cases [3]. $80 \%$ tumors occur bilaterally [4].

Average age of patients with this tumor is 45 years and is more common in young age. In addition ascites is a common presentation in Krukenberg tumor and usually reveals malignant cells. However a case has been reported of bilateral
Krukenberg tumor with ascites and right sided hydrothorax that revealed no malignant cells [5]. This is called pseudo Meig's syndrome in contrast to Meig's syndrome associated with a triad of benign ovarian tumor, ascites and right sided hydrothorax. Patients usually present with symptoms related to ovarian involvement such as abdominal pain and distension. Less commonly patients may also present with vague gastrointestinal symptoms.

Present case is unusual in that initial presentation of colon malignancy was a gynecological emergency in form of acute abdomen due to torsion and rupture of right sided ovarian mass. Ovarian torsion is the twisting of an ovary on its ligamentous or adenexal support or both. Concomitant tubal and ovarian torsion is also seen in upto $67 \%$ of the cases of adenexal torsion. Ultrasound is the initial and easily available modality in any setup to diagnose torsion ovary and shows interruption of blood supply. Currently in literature there is no reported cases of Krukenberg tumor presenting to gynecological emergency as acute ovarian torsion and rupture. Therefore, in addition to benign neoplasms which are more common in this age group [6], less common Krukenberg tumor must also be considered for differential diagnosis in such a scenario. Krukenberg tumor carries an extremely gloomy prognosis and average survival time ranges between 3-10 months. Only 10\% patients may survive for 2 years [7].

Acknowledgement: I acknowledge Dr Punam Bajaj, Joint Director, NABL, Quality Council of India for her immense help and personal inputs as pathologist.

Contributors: PL participated in the patient care, and manuscript writing. KA and AS participated in the patient care, manuscript editing. RL participated in surgical management and revising the manuscript critically. PL will act as a study guarantor. All authors approved the final version of this manuscript.

Funding: None; Competing interests: None stated.

\section{References}

1. Al-Agha OM, Niscastri AD. An in-depth look at Krukenberg tumor: Archives of Pathology \& Laboratory Medicine. 2006;130:1725-1730. 
2. Young RH. From Krukenburg to today: the ever present problems posed by metastatic tumors in the ovary: part 1. Historical perspective, general principles, mucinous tumors including the Krukenburg tumor. Adv Anat Pathol. 2006;13:205-227.

3. Holds F, Hart WR Krukenberg tumors of the ovary: A clinicopathologic analysis of 27 cases. Cancer. 1982;50:2438-2447.

4. McGill FM, Ritter DB, Rickard CS, Kaleya RN, Wadler S, Greston WM, et al. Krukenberg tumors: Can management be improved? Gynecol Obstet Invest.
1999;48:61-65.

5. Cetin B, Aslan S, Akinsi M, Atalay C, Cetin A. Along surviving case of pseudomeig's syndrome caused by Krukenberg tumor of the stomach. Jpn J Clin Oncol. 2005;35:221-223.

6. Chang HC, Bhatt S, Dogra VS. Pearls \& pitfalls of ovarian torsion. Radiographics. 2008;28:1355.

7. Yook JH, Ch ST, Kim BS. Clinical prognostic factors for ovarian metastasis in women with gastric cancer. Hepatogastroenterology. 2007;54:955-959. 